\title{
A Mechanism for Propulsion without The Reactive Ejection of Matter or Energy
}

\author{
Remi Cornwall \\ University of London Alumni Society, Malet Street, London WC1E 7HU \\ http://webspace.qmul.ac.uk/rocornwall http://ulondon.academia.edu/RemiCornwall
}

\begin{abstract}
This paper updates earlier thoughts by the author on a putative propulsion system. The concept was based around static electromagnetic momentum, as expounded in the "Feynman Disk" and experimentally verified by Graham and Lahoz. That said, naïve static electromagnetic momentum schemes to achieve linear translation are defeated by "hidden momentum" mechanisms, so too are simple arrangements just cycling the fields; we shall survey the flaws in their arguments. It may however be possible to achieve linear translation by means of arrangements of torques with a novel mechanism to break the symmetry of forces (or torques) on the second half of the cycle as the field is switched off. At the time of earlier presentation no mechanism could be found to explain the momentum balance for the process but it was believed that momentum was being given to the zeropoint of the field. We show that it is possible to dump angular momentum and thence linear momentum to the ground state by standard quantum analysis of the EM field. None of this violates the conservation of momenergy.
\end{abstract}

\section{Introduction}

All propulsion or motive means obey the conservation of momentum. Momentum (and angular momentum) is conserved for a good reason: it represents the homogeneity and isotropy of space, a symmetry constraint like all conservation laws[1]. Put simply, if one was running around a track (and wasn't tired) but found the time for one leg of the circuit quicker than the other, one would conclude that the track wasn't "fair", one leg of it must have a head-wind or a gradient; it wouldn't be a symmetric track. Similarly, if we inserted a spinning top into a fairly tenuous fluid and observed that attempts to spin it one way were easier than the reverse way, we would conclude that the two rotation directions were not symmetrical. The free space of rocket travel offers no such free lunch, no preferred directions.

Relativity theory subsumes, by the transformation of frames, this into one concept of the Conservation of Momenergy[2-4]. One might consider motion with respect to a ponderable body by being in direct contact with it and pushing against it and then coming back to rest, some distance away, by pushing again in the reverse sense. None of these steps violates the Principle of Conservation of Momenergy.

If we observe momentum not being conserved, we would automatically break the $1^{\text {st }}$ Law of Thermodynamics too, seen from another frame in relative motion. Violation of the $1^{\text {st }}$ Law would entail the creation (or destruction) of energy or matter out of nothing (or to nothing). Turning away from probably the more metaphysical aspects of Cosmology, on the level of theoretical and applied physics or engineering science, the $1^{\text {st }}$ Law is very sacrosanct. This is said in an understated manner. Science is defined as the systematic acquisition and categorisation of knowledge. Science, as well as being the result of billions upon billions of personhours of observation and theoretical musings, must have its strong foundations.

The "miracle" of rocket propulsion in vacuo requires the expulsion of reactive mass or energy counter to the direction of motion (the $1^{\text {st }}$ Law is not violated when the complete system of rocket and expelled mass is considered). It becomes necessary for a craft to carry this reactive mass and a measure of its "efficiency" in doing this is the Specific Impulse[5] as a ratio of thrust to propellant flow rate:

$$
I_{s p}=\frac{\text { Thrust }}{\dot{m}}
$$

The units are velocity if Newton/Kilogram/s is used or seconds if thrust is measured in Newtons and the propellant flow rate is measured as weight per second. Propeller, turboprops and jet-engined aircraft have high specific impulses because most of their reaction mass in not carried in the craft (as the fuel/oxidizer) but present in the air they fly through. Rockets, of course, fare much worse with the expression (eqn. 1) reducing to:

$$
\begin{aligned}
& I_{s p}=\dot{m} v_{\text {exhaust }} / \dot{m}=v_{\text {exhaust }} \\
& \text { or } \\
& I_{s p \text { seconds }}=\frac{\Delta p_{\text {exhaust }}}{\Delta m g}=\frac{v_{\text {exhaust }}}{g}
\end{aligned}
$$


The expression above uses the second definition of specific impulse to work in the factor of ' $g$ ', the acceleration due to gravity, which is most pertinent to rocketry and interplanetary space travel.

Ultimately specific impulse gives a measure of how fast one can go (or even how far, if one is subject to a resistive force) for a given amount of fuel and oxidizer contained in the tanks. The limit to space craft travel is not so much the energy (or more correctly, the enthalpy) liberated by the fuel/oxidizer but the reactive mass, one could contend with having a rocket somehow nuclear powered. Should we convert some of the energy liberated by nuclear power into mass or emit all the energy liberated rearward as photons? Simple Relativity theory[2, 4] provides us the answer by the basic dispersal relation/rest-mass invariance relation:

$$
E^{2}-\mathbf{p}^{2} c^{2}=m^{2} c^{4}
$$

This is at the root of the variation of energy and momentum of a body with velocity:

$$
\begin{aligned}
& E=\frac{m c^{2}}{\sqrt{1-\frac{v^{2}}{c^{2}}}} \\
& \mathbf{p}=\frac{m \mathbf{v}}{\sqrt{1-\frac{v^{2}}{c^{2}}}}
\end{aligned}
$$

Dividing eqn. 5 by eqn. 4 gives us a measure of specific impulse:

$$
\frac{\mathbf{p}}{E}=\frac{\mathbf{v}}{c^{2}}
$$

Whereupon the answer to our question is solved: our nuclear powered rocket gains the greatest specific impulse by emitting photons. The "exchange rate" for this is some $1 \mathrm{~N}$ of force per $300 \mathrm{MW}$ of power generated. These notions rule out half-considered nonsense conjecture of conjuring matter out of the vacuum (pair-creation[6]) between two charged plates (or similar) and then accelerating it[7]; eqn. 4 contains the complete energy budget and it is clear that the step of creating matter is superfluous - just expel photons. The universe has been generous to us and if we take the availability of matter as a given (so we do not have to expend energy creating it, that was done for us at the Big-Bang), we could contend with electromagnetic propulsion by the expulsion of ions at high velocity from a particle accelerator. The specific impulse then would be:

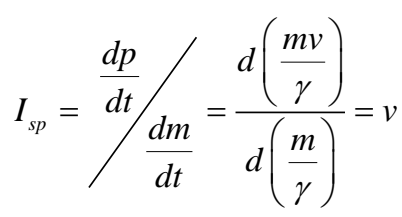

Where the relativistic correction term in eqn. 4 and eqn. 5 is represented by $\gamma$. Thus it can be seen that Ion Propulsion[5] gives the highest specific impulse, though practically thrusts are very small: one mole of matter requires an integer multiple of a Faraday of electrical charge (fundamental charge multiplied by Avogadro's constant), which is huge. Even a mole of the heaviest naturally occurring element, uranium is of the order of a few hundred grams. Taken that it may be accelerated to high speed, some 96500 Coulombs of charge per second (96500 Amperes) is required per mole of charge. If $238 \mathrm{~g} / \mathrm{s}$ of mono-charged uranium was expelled at $10 \mathrm{KeV}$ per particle, that would require nearly $1 \mathrm{GW}$ of power, with an exhaust velocity of $90 \mathrm{~km} / \mathrm{s}$ and a thrust of some $11 \mathrm{kN}$ (power / velocity).

That is quite an engineering challenge. However that thrust figure is comparable to jet and rocket engine thrusts; also nuclear power plants can be quite compact: aboard a nuclear submarine, some 100s of megawatts of power is generated in a volume of a few cubic meters. There would be considerable additional volume required for auxiliary equipment, of which a large part would definitely be the particle accelerator. It is interesting to note that calutrons (essentially particle accelerators) used for isotope separation are massive and deliver microgram quantities after running for several hours.

To conclude this introduction, all propulsion must be reactive; conservation of momentum is wellfounded and "sacrosanct". Specific impulse gives a good measure of performance of a propellant carrying reactive system and with this viewpoint, photon rockets or ion-engines are the best we can do; both have poor thrust due to current engineering and material science limitations.

We ask: can we do better? Is there any other phenomenon we could utilise for propulsion, other than by reactive means? In the next section we shall look at the phenomenon of hidden static electromagnetic momentum.

\section{Hidden static momentum defeats naïve linear thrusters}

Conservation of energy allows us to set up a continuity equation for electromagnetic energy in one region to flow and do work against a cloud of charges[2-4]: 


$$
-\frac{\partial u}{\partial t}=\nabla \cdot \mathbf{S}+\mathbf{F} \cdot \mathbf{v} \quad \text { eqn. } 8
$$

This simply states that the loss of the energy density of the electromagnetic field, $u$, per unit time is equal to the outward flow of the differential energy flux $\mathbf{S}$ (the Poynting vector) and the work done by the force density against the cloud of charges moving at velocity v. Written in its full glory with the energy density and Lorentz force density, we obtain:

$$
\begin{aligned}
-\frac{\partial}{\partial t} \frac{\varepsilon_{0}}{2}\left(\mathbf{E}^{2}+c^{2} \mathbf{B}^{2}\right) & =\nabla \cdot \mathbf{S}+\rho(\mathbf{E}+\mathbf{v} \times \mathbf{B}) \cdot \mathbf{v} \\
\Rightarrow-\frac{\partial u}{\partial t} & =\nabla \cdot \mathbf{S}+\mathbf{J} \cdot \mathbf{E}
\end{aligned}
$$

In the last step, the current density $\mathbf{J}$ has been identified. It is then an easy matter by vector manipulation to identify the Poynting vector, which is the flow of electromagnetic field energy:

$$
\mathbf{S}=\varepsilon_{0} c^{2} \mathbf{E} \times \mathbf{B}
$$

The electromagnetic momentum flux density will be this figure divided by $\mathrm{c}^{2}$ as a natural consequence of Relativity[3-4]:

$$
\mathbf{g}=\varepsilon_{0} \mathbf{E} \times \mathbf{B}
$$

In the dynamic case of varying $\mathrm{E}$ and $\mathrm{B}$ fields associated with wave transport, this explains phenomenon of radiation pressure. However, if taken literally, static sources of crossed electric and magnetic fields would have associated with them a flow of energy and momentum, Feynman pointed this out in his disk paradox[2].

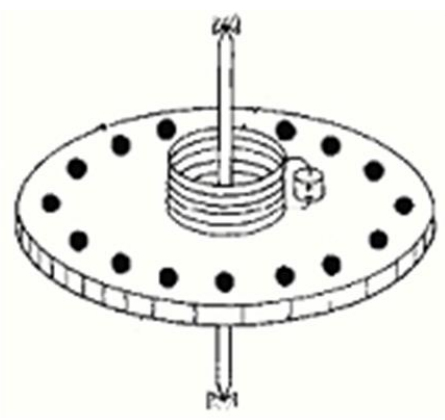

\section{Figure 1 - Feynman Disk Paradox}

In the figure the radial electric field from charged balls is crossed upon the solenoidal field from the coil on the disk's axis, with the implication that there is a flow of momentum around the disk. Granted too, when the solenoid is switched on, the changing magnetic field (or vector potential) will induce a circumferential electric field, which must react against the charged balls; the disk would rotate. The mechanical aspect of the setup has reacted against the electromagnetic field. Upon switching off the solenoidal field, the disk will stop rotating (this discounts friction). Momentum has been conserved. Fantastical as this sounds, this has been experimentally verified by Graham and Lahoz[8].

Naturally thought turned to generating linear thrust and figure 2 shows the essence of a naïve thruster, which is defeated by the hidden momentum argument[9-11]. The basic outline of this argument is that, there is hidden mechanical momentum from the flow of charge carriers that generate the magnetic field. It is a Relativistic effect from the charge carriers having more energy (and hence more mass) from their potential in the electric field - what is neglected in a simple analysis, is the effect of pressure on the stress-energy tensor of the fluid that constitutes the charge carriers. This just cancels any linear momentum generated by the field.

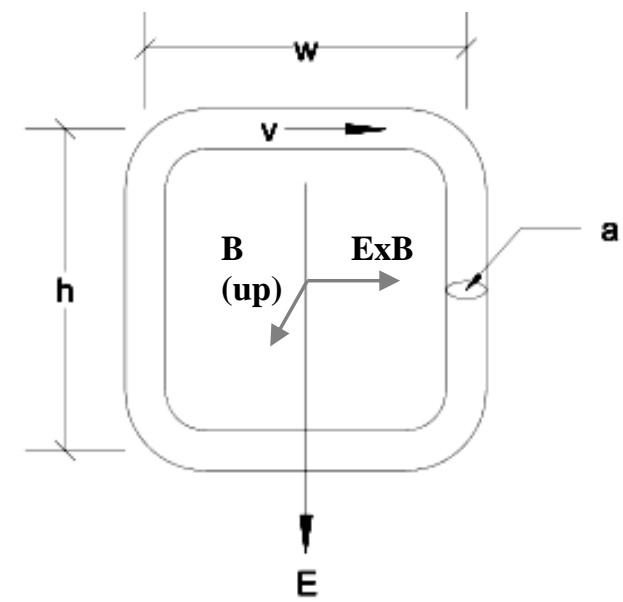

Figure 2 - Naïve linear ExB thruster and the hidden momentum argument against it

The feature of the current argument can be seen in figure 2 above, where the (negative) charge carriers moving with velocity, $\mathrm{v}$, on the lower leg of the circuit are at higher potential from the electric field, E. Puthoff[12] (in the appendix 1 of said paper) gives a quick satisfactory proof to dispel these types of device without much further analysis.

\subsection{Naïve cycling of the fields doesn't work either}

The hidden momentum argument prevents simple static schemes from generating linear momentum, so perhaps we might try cycling the fields (with reference to figure 1), so that on the latter half of the cycle the E-field generated by the coil's falling flux might be cancelled. Consider the average momentum over a cycle of length $\mathrm{T}$ units of time:

$$
\langle\mathbf{p}\rangle=\frac{1}{T} \int_{T}\left[\varepsilon_{0} \int_{V} \mathbf{E} \times \mathbf{B} d V\right] d t
$$


The force is the time derivative of the momentum and so the average force can be computed,

$$
\begin{aligned}
& \langle\mathbf{f}\rangle=\frac{1}{T} \int_{T}\left[\frac{d}{d t} \varepsilon_{0} \int_{V} \mathbf{E} \times \mathbf{B} d V\right] d t \\
& \Rightarrow \\
& \langle\mathbf{f}\rangle=\frac{\varepsilon_{0}}{T}\left\{\left[\int_{V} \mathbf{E}_{t=T} \times \mathbf{B}_{t=T} d V\right]-\left[\int_{V} \mathbf{E}_{t=0} \times \mathbf{B}_{t=0} d V\right]\right\} \\
& =0
\end{aligned}
$$

Over a cycle less than the time it takes for the fields to propagate, any way the E and B fields are cycled leads to the same boundary conditions at the cycle start and end points and thus the net force generated is zero. We shall seek a way around this argument by first reviewing the experimental fact of the Feynman Disk/Graham-Lahoz experiment and seeking deeper understanding about it.

\section{A mechanism for the Graham-Lahoz experiment}

Although we have dismissed linear static ExB momentum schemes, the generation of angular momentum (figure 1) is permitted [2] and has been experimentally verified[8]. The Feynman disk setup allows angular translation by the generation of a torque of the disk (the balance being taken by the ExB contribution of the fields) when the coil is switched on and then the reverse torque when the coil is switched off. A similar argument to eqn. 13 shows that over a cycle there is no net torque but angular translation is permitted. In their paper, Graham and Lahoz wrote:

\footnotetext{
"It is remarkable that no known 'particle' can be identified as the agent of the observed electromagnetic angular momentum in the exchange with the mechanical detector. However, this does not imply that a new entity has to be introduced, because the concept of energymomentum carried by macroscopic quasistatic electromagnetic field is already contained in Maxwell's equations. According to these, and as directly implied by our experimental result, permanent magnets and electrets can be used to build a flywheel of electromagnetic energy steadily flowing in circles in the vacuum gap of a capacitor as if Maxwell's medium were endowed with a property corresponding to super-fluidity. The certainly new insight is that the quasi-static Maxwell's field is not merely an unobservable medium of interaction between matter and matter: it has in fact the mechanical properties postulated by Maxwell, in contradistinction to any 'action at a distance' theory."
}

So there is a problem: the spatially static flow of energy is not a wave phenomenon and cannot be described by a photon flux. In the limit of zero frequency all electromagnetic phenomena become classical - any Fourier expansion of the field would tend to zero frequency. However the second quantisation of the electromagnetic field (appendix 1 and 2) can surprisingly explain the result within the context of quantum electrodynamics.

Appendix 2 developed the expression for the Poynting vector and the momentum flux of photons:

$$
\mathbf{p}=\sum_{k} \hbar \mathbf{k}\left(a^{\dagger} a+\frac{1}{2}\right)
$$

Normally a photon is represented by a Gaussian wave packet, as the envelope is self-similar through the Fourier transform process, which defines conjugate position/momentum pairs in quantum mechanics and the Heisenberg Uncertainty relations. It is of note that the double-sided Fourier transform is used, which covers both positive and negative $\mathrm{k}$-values; the effect of this is that the $1 / 2$ "zeropoint" contribution is cancelled.

We argue that this symmetry is broken in the Feynman disk setup; the spatial constraints of the system do not allow negative wavevector values. The photons could only be described in this arrangement by a single-sided Fourier transform. Let us consider why this is so with reference to figure 1 . If the coil is on and a steady magnetic field is present, then we know[2-3],

$$
\begin{aligned}
& \mathbf{B}=\operatorname{curl} \mathbf{A} \\
& \Rightarrow \int_{C} \mid \mathbf{A} \cdot d \mathbf{l}=\int_{\text {Inside } C} \mathbf{B} \cdot \mathbf{n} d a
\end{aligned}
$$

The vector potential takes the form,

$$
\mathbf{A}=\frac{1}{2} \mathbf{B} \times \mathbf{r}
$$

This is clearly oriented in one direction around the coil, as the coil's flux is unequivocally up or down. Then the E-field acting on the charged balls on the peripheral of the disk (figure 1) is given by[2-3]:

$$
\mathbf{E}=-\frac{\partial \mathbf{A}}{\partial t}
$$

Subsequent Fourier expansion of the field (appendix 1) would only contain positive frequency components to represent a "phasor" turning in the complex plane.

The upshot of this is that photons in this case are inescapably coupled to their ground state and momentum considerations for the system need to consider both. Thus when the electrodynamics has 
died down and the system becomes quasi-static, the flow of momentum has been transferred to the zero-point of the harmonic oscillators that were involved in the initial photon flux. When the fields cycle again, the reverse coupling from the zeropoint to the photon fields occurs.

\section{A means to generate net angular momentum without the expulsion of reactive momentum}

Returning to the cycling argument, we can now justify angular momentum being dumped to the zero-point in a cyclical process from the proceeding section. Considering the average angular momentum around a cycle:

$$
\langle\mathbf{L}\rangle=\frac{1}{T} \int_{T}\left[\varepsilon_{0} \int_{V} \mathbf{r} \times \mathbf{E} \times \mathbf{B} d V\right] d t \quad \text { eqn. } 16
$$

To be considered a cycle, E and B must obviously return to their initial values and we calculate the average torque:

$$
\begin{aligned}
& \langle\boldsymbol{\tau}\rangle=\frac{1}{T} \int_{T}\left[\frac{d}{d t} \varepsilon_{0} \int_{V} \mathbf{r} \times \mathbf{E} \times \mathbf{B} d V\right] d t \\
& \Rightarrow\langle\boldsymbol{\tau}\rangle=\frac{\varepsilon_{0}}{T}\left\{\begin{array}{l}
+\left[\int_{V} \mathbf{r}_{\text {final }} \times \mathbf{E}_{t \rightarrow T} \times \mathbf{B}_{t \rightarrow T} d V\right] \\
-\left[\int_{V} \mathbf{r}_{\text {initial }} \times \mathbf{E}_{t=0} \times \mathbf{B}_{t=0} d V\right]
\end{array}\right\} \text { eqn. } 17
\end{aligned}
$$

The point is, as the fields return to their initial value, the radius, $\mathbf{r}$, doesn't need to be kept constant and the average torque isn't zero. The previous section showed that it is permissible to dump angular momentum to the zero-point/groundstate of the field. Figure 3 shows how this can be practically implemented.

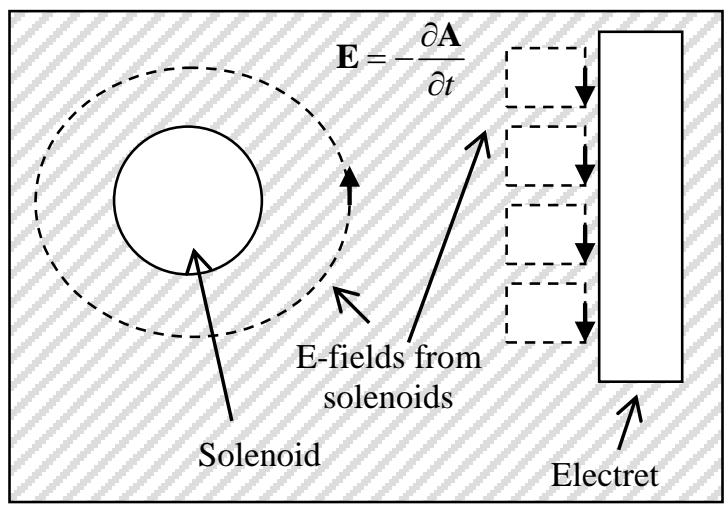

Figure 3-Method of cancelling fields on the second half of the cycle

The figure shows, all bolted onto the same plate, the main solenoid to the left, a group of smaller cancellation solenoids to the right and to the left of that, the electret (permanently electrically charged material). As by the argument of eqn. 13, the cancellation solenoids will have no effect in cycling in generating linear momentum but by eqn. 17, they can cancel the E-field from the large solenoid and do so at a different radius, so that the whole arrangement leaves the electret with angular momentum and the balance of the angular momentum left with the ground state.

If we include all systems: the mechanical, the photon field, the zero-point, then angular momentum and energy is conserved but more shall be said on this at the end of the next section (5.1).

\section{A means to generate net linear momentum without the expulsion of reactive momentum}

Mirror duplication of the arrangement of figure 3 allows for linear force to be generated. If a torque or couple is the application of force off-centre of the force, one merely needs to mirror duplicate to resolve a force that moves both centres (figure 4). The arrangement is akin to a conveyor belt moved between counter-rotating rollers. Obviously not all the forces arising from the too-and-fro of electromagnetic energy will resolve in the direction we require and they will just elastically deform the plate.

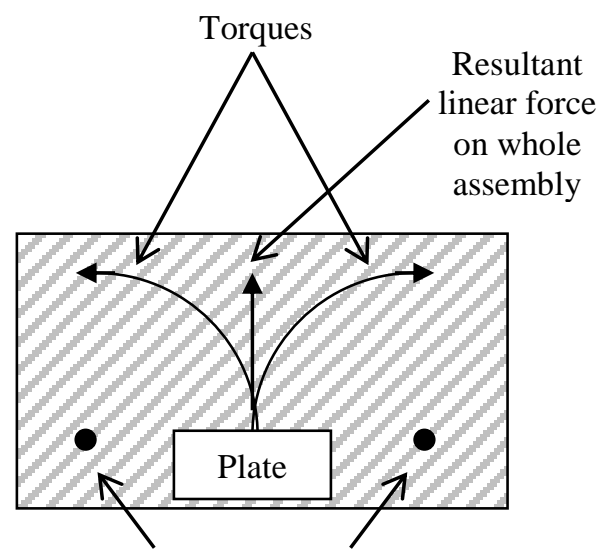

Centre of turning effect

Figure 4 - Resolution of torques into a linear force

A rough estimate of the forces follows from eqn. 17 ,

$$
f \propto \frac{\varepsilon_{0} B E V}{T}
$$

This warrants another paper on the engineering of such a scheme, suffice to say that high frequency cycling by, perhaps, a microwave or optical cavity would bring down the cycle time T. We shall try a rough estimate of the forces by looking at the materials and methods: we could choose to use an electret and solenoids or the electrical dual, a magnet and e-field generator. 
Let us consider a microwave cavity with a ferromagnet:

$$
\begin{aligned}
& \mathrm{B} \sim 1 \text { Tesla } \\
& \mathrm{E} \sim 3 \times 10^{4} \mathrm{~V} / \mathrm{m} \text { in air } \\
& 1 / \mathrm{T} \sim 10 \mathrm{GHz}
\end{aligned}
$$

The force would be the order of $2650 \mathrm{~N}$ per $\mathrm{m}^{3}$ of electret/ferromagnet. We might consider some kind of optical cavity in a vacuum with powerful lasers and electric field generators (i.e. the reverse of electret/ferromagnet):

$$
\begin{gathered}
\mathrm{B} \sim 10 \text { Tesla (estimate from power and } \\
\text { Poynting expression for a } \\
\text { modestly powerful laser) } \\
\mathrm{E} \sim 10^{4} \mathrm{~V} / \mathrm{m} \text { from E-field generator } \\
1 / \mathrm{T} \sim 10^{14} \mathrm{~Hz}
\end{gathered}
$$

And the force is some $90 \mathrm{MN}$ per $\mathrm{m}^{3}$ of electret/ferromagnet, though this would obviously be de-rated for material science concerns. Rough engineering formulas be damned - if we compare the thrust of the mighty Saturn V rocket, some $3.4 \mathrm{MN}$ - but eqn. 18 shows the possibilities if the basic physics is correct... Much as splitting a few atoms definitely showed a small, though relatively fantastic amount of heat production (the basic physics showed the equivalence of mass-energy), the ensuing scale-up to the A-bomb, H-bomb or even peaceful power production took a cast of tens of thousands and a huge budget too.

\section{$\underline{5.1}$ Momenergy is conserved}

It was stressed in section 3, appendices 1 and 2 that in considering the quasi-static ExB angular momentum that the zero-point of the field was coupled to the photon momentum operator (eqn. 31 appendix 2) due to the wavevectors being unipolar; the whole system: mechanical, fields and zeropoint need to be considered. Section 4 and eqn. 17 showed that angular momentum could be dumped to the ground state of the harmonic oscillators of the radiation field that set up the static momentum. This has the effect of rotating the whole EM field which has massive energy (including the zeropoint); obviously with its huge mass the rotation will be infinitesimal.

Consider now a trip between two space stations which are very large, relative to our craft but not so large as to exert a significant gravitational force on it. The propulsion system pushes directly off the space stations by a rod and the craft does a certain amount of work doing this. The co-ordinate system shall be with respect to the first station.

The craft pushes off and has a certain amount of kinetic energy that allows it to coast to the second station, which moves at some relative speed to the first station. The craft matches the second station's speed and alights on it. The return trip does the reverse and once again, the craft does a certain amount of work pushing off.

The mass of the stations is very large compared to the craft and hardly any change occurs in their velocities. Once returned, the craft's computers say that a certain amount of fuel has been used and a certain amount of work done. Back in the coordinate system of the first station it would appear that energy has gone missing - work was done by the propulsion system and yet it has no kinetic energy. A detailed analysis taking into account the slight change in velocity of the stations would, of course, show on the round trip that this work has been re-partitioned to the stations' kinetic energy, where in a sense it is hidden from the craft's future missions (it can't be reclaimed and the next mission will require slightly more work by the propulsion system).

The core argument about the dumping of angular momentum and the mirroring scheme (figure 4) to generate linear momentum is very analogous to the story of the craft between the two space stations. A craft based on a linear propulsion device expounded herein will have its work and kinetic energy, as it moves between frames, re-partitioned to the ground state/zero-point of the electromagnetic field; indeed with the many more degrees of freedom of the field, this is just the equipartition of energy. In a sense, the whole electromagnetic field has been set "moving", though with its unimaginable mass, that movement is infinitesimal, though the energy and momentum changes are finite.

\section{Conclusion}

Classical (quasi-static) electromagnetic angular momentum exists and is proven by experiment and theory.

It is believed that this paper has provided a mechanism for the classical quasi-static observation of a flow of energy that can't be explained by radiation (particles/photons) since it is a zero frequency effect, yet counter-intuitively it depends on quantum effects of the ground state of the field.

Simple linear momentum from ExB is balanced by hidden momentum, a relativistic effect. In the angular momentum ExB case (which is experimentally verified, to repeat and stress), wavevectors describing the photon field can only be unipolar. This inescapably leads to the requirement of considering the zero-point of the 
radiation fields setting up the $\mathrm{E}$ and $\mathrm{B}$ fields; one must consider both for the photon momentum operator.

The considering of the ground-state/zero-point as another system capable of receiving momentum and that the whole system: mechanical, radiation and zero-point needs to be considered, offered the intriguing prospect of dumping momentum to the ubiquitous (and massive) ground state of the electromagnetic field.

We presented an argument for a cyclical process where the radius is varied in the expression for the angular electromagnetic momentum. This appears to allow for the field to be cycled but leaving net angular momentum to the mechanical system; the total angular momentum is balanced if we take into account the zero-point of the field. An arrangement of two such devices generating angular momentum was then able to generate linear momentum. The basic physics suggests that this is possible.

\section{Appendix 1 Quantisation of the Electromagnetic} Field

The simple harmonic oscillator provides the prototype for particle physics and the quantisation of fields[6]. We can write the Hamiltonian operator[13] of a particle with a linear spring as:

$$
\hat{H}=\frac{\hat{p}^{2}}{2 m}+\frac{1}{2} k \hat{x}^{2}
$$

The position and momentum operators can be related to the "ladder" creation and annihilation operators[13],

$$
\begin{aligned}
& a^{\dagger}=\sqrt{\frac{m \omega}{2 \hbar}\left(\hat{x}-\frac{i}{m \omega} \hat{p}\right)} \\
& a=\sqrt{\frac{m \omega}{2 \hbar}\left(\hat{x}+\frac{i}{m \omega} \hat{p}\right)}
\end{aligned}
$$

(If we put $\omega=\sqrt{\frac{k}{m}}$ )

So that,

$$
\begin{aligned}
& \hat{x}=\sqrt{\frac{\hbar}{2 m \omega}}\left(a^{\dagger}+a\right) \\
& \hat{p}=\sqrt{\frac{\hbar m \omega}{2}}\left(a^{\dagger}-a\right)
\end{aligned}
$$

By which we can write an alternative form for the oscillator,

$$
\begin{aligned}
& \hat{H}=\frac{1}{2} \hbar \omega\left(a^{\dagger} a+a a^{\dagger}\right) \\
& =\hbar \omega\left(a^{\dagger} a+\frac{1}{2}\right)
\end{aligned}
$$

For which we know, classically at least, that the solutions is sinusoidal and the momentum and displacement form quadrature pairs, for the differential relation between them. By fairly loose analogy that the Hamiltonian is the total energy of the system and that the E and B fields are quadrature pairs, by classical electromagnetism too we can intuit the corresponding quantum Hamiltonian operator:

$$
\hat{H}=\frac{\varepsilon_{0}}{2} \int_{V}\left(\hat{E}^{2}(\mathbf{r}, t)+c^{2} \hat{B}^{2}(\mathbf{r}, t)\right) d^{3} \mathbf{r} \quad \text { eqn. } 23
$$

Considering the solution of the classical EM wave equation as a transverse field, we can write the vector potential solution as a Fourier expansion in a cubic box of volume V (we leave out the polarisation to streamline the discussion and get to the point),

$$
\mathbf{A}(\mathbf{r}, t)=\sum_{\mathbf{k}}\left(a_{\mathbf{k}}(t) e^{i \mathbf{k} \cdot \mathbf{r}} e^{-i \omega t}+a_{\mathbf{k}}^{*}(t) e^{-i \mathbf{k} \cdot \mathbf{r}} e^{i \omega t}\right) \text { eqn. } 24
$$

Where $\mathbf{k}$ is the wavevector, $\omega$ is the frequency and $a$ and its complex conjugate $a^{*}$ are the Fourier coefficients. Electrodynamics give us the following relations between the $\mathrm{E}, \mathrm{B}$ and the potentials:

$$
\begin{aligned}
& \mathbf{B}=\nabla \times \mathbf{A} \\
& \mathbf{E}=-\nabla \phi-\frac{\partial \mathbf{A}}{\partial t}
\end{aligned}
$$

From which we can calculate the fields in terms of the Fourier expansion:

$$
\begin{aligned}
& \mathbf{E}(\mathbf{r}, t)=i \omega \sum_{\mathbf{k}} a_{\mathbf{k}}(t) e^{i \mathbf{k} \cdot \mathbf{r}} e^{-i \omega t}-a_{\mathbf{k}}^{*}(t) e^{-i \mathbf{k} \cdot \mathbf{r}} e^{i \omega t} \\
& \mathbf{B}(\mathbf{r}, t)=i \sum_{\mathbf{k}} a_{\mathbf{k}}(t) e^{i \mathbf{k} \cdot \mathbf{r}} e^{-i \omega t}-a_{\mathbf{k}}^{*}(t) e^{-i \mathbf{k} \cdot \mathbf{r}} e^{i \omega t}
\end{aligned}
$$

(The orientation of the $\mathbf{B}$ vector relative to $\mathbf{E}$ isn't shown for brevity)

Squaring the expressions in eqn. 26 (and taking note of orthogonality conditions) and substituting into the classical EM Hamiltonian we obtain:

$$
\begin{aligned}
& H=\frac{\varepsilon_{0}}{2} \int_{V}\left(E^{2}(\mathbf{r}, t)+c^{2} B^{2}(\mathbf{r}, t)\right) d^{3} \mathbf{r} \\
& \Rightarrow V \varepsilon_{0} \sum_{\mathbf{k}} \omega^{2}\left(a_{\mathbf{k}}^{*}(t) a_{\mathbf{k}}(t)+a_{\mathbf{k}}(t) a_{\mathbf{k}}^{*}(t)\right)
\end{aligned}
$$

Now by analogy to the QM harmonic oscillator we identify, by eqn. 22 and eqn. 23, the Fourier modes 
of the EM wave to the creation and annihilation operators (and with use of the boson commutation relations) thus,

$$
\begin{aligned}
& a_{k}(t) \rightarrow \sqrt{\frac{\hbar}{2 \omega V \varepsilon_{0}}} a(\mathbf{k}) \\
& a_{k}^{*}(t) \rightarrow \sqrt{\frac{\hbar}{2 \omega V \varepsilon_{0}}} a^{\dagger}(\mathbf{k})
\end{aligned}
$$

And the corresponding Hamiltonian for the independent oscillators of the field is:

$$
\begin{aligned}
& H=\frac{1}{2} \sum_{k} \hbar \omega\left(a^{\dagger} a+a a^{\dagger}\right) \\
& \text { or } \\
& H=\sum_{k} \hbar \omega\left(a^{\dagger} a+\frac{1}{2}\right)
\end{aligned}
$$

The $1 / 2$ signifies the zeropoint energy, which when summed over all the modes up to the Planck frequency indicates a very high energy density. This large energy density is not normally observed as observables generally result from the differentiation of a potential (gauge theory), though several phenomena in physics are attributed to its residual effects via perturbation theory (Lamb Shift, Van der Waal/London/Casimir Forces[14]).

\section{Appendix 2 Quantisation of Photon Momentum}

In the main text the classical photon momentum density was given by the Poynting expression (eqn. 11),

$$
\mathbf{g}=\varepsilon_{0} \mathbf{E} \times \mathbf{B}
$$

We can integrate this over the volume, $\mathrm{V}$, of the box (appendix 1) and relate the classical field quantities to the modes of the Fourier expansion of the field for the momentum:

$$
\mathbf{p}=V \varepsilon_{0} \sum_{\mathbf{k}} \omega \mathbf{k}\left(a_{\mathbf{k}}^{*}(t) a_{\mathbf{k}}(t)+a_{\mathbf{k}}(t) a_{\mathbf{k}}^{*}(t)\right) \quad \text { eqn. } 30
$$

This can then be easily related to the creation and annihilation operators:

$$
\mathbf{p}=\sum_{k} \hbar \mathbf{k}\left(a^{\dagger} a+\frac{1}{2}\right) \quad \text { eqn. } 31
$$

Normally one would sum over positive and negative $\mathrm{k}$ and the $1 / 2$ zeropoint term would cancel. The point of this paper argues in the main text that if $\mathrm{k}$ is not bi-directional (as by the static angular momentum in the Feynman disk setup circling in one direction), that the zeropoint term should be included when describing the whole system.

One further point needs to be made too, static electromagnetic momentum is a strictly classical effect[8] where the frequency tends to zero. However there are no infinite time processes and the fields must be established, thus the analysis and point we make is relevant.

\section{$\underline{\text { References }}$}

1. Landau Lifshitz, A Course in Theoretical Physics: Mechanics. Vol. 1. 1982: Butterworth Heinemann.

2. Feynman Leighton Sands, The Feynman Lectures on Physics. Addison-Wesley, Reading, Massachusetts. Vol. 21989.

3. Jackson, J.D., Classical Electrodynamics. 2nd ed. 1975: Wiley.

4. Landau Lifshitz, A Course in Theoretical Physics: Classical Theory of Fields. Vol. 2 1982: Butterworth-Heinemann.

5. Sutton Biblarz, Rocket Propulsion Elements. 9th ed. 2017: Wiley.

6. Landau Lifshitz, A Course in Theoretical Physics: Quantum Electrodynamics. Butterworth-Heinemann. Vol. 4. 1984.

7. White March Lawerence et al, Measurement of Impulsive Thrust from a Closed Radio-Frequency Cavity in Vacuum. Journal of Propulsion and Power, 2016.

8. Graham Lahoz, Observation of static electromagnetic angular momentum in vacuo. Nature, 1980. 285(154).

9. Hernandez, A., The Feynman paradox revisited. Eur. J. Phys., 1981. 2: p. 168170.

10. Comay, Exposing "hidden momentum". Am. J. Phys., 1996. 64(8).

11. Kirk, T. On the Definition of "Hidden" Momentum. 2017; Available from: http://physics.princeton.edu/ mcdonald/ex amples/hiddendef.pdf.

12. Puthoff, Engineering the Zero-Point Field and Polarizable Vacuum for Interstellar 
$-9-$

Flight. Journal of the British

Interplanetary Society, 2001.

13. Landau, L., A Course in Theoretical

Physics: Quantum Mechanics. Vol. 3.

1982: Butterworth-Heinemann.

14. Landau, L., A Course in Theoretical

Physics: Quantum Electrodynamics.

Butterworth-Heinemann. Vol. 4. 1984. 